\title{
HILAIRE BELLOC: DEL ESTADO DEL BIENESTAR AL ESTADO SERVIL
}

\author{
ALFONSO DÍAZ VERA"
}

Fecha de recepción: 4 de marzo de 2016

Fecha de aceptación: 12 de diciembre de 2016

Resumen: Hilaire Belloc (1870-1953) criticó la legislación social del Partido Liberal en la Inglaterra de los años anteriores a la Primera Guerra Mundial, considerada precedente del Estado del Bienestar. Su crítica se basó en una línea de pensamiento conocida como distributismo, que, fundamentándose en una filosofía de raíz tomista y tomando como punto de partida la encíclica Rerum Novarum, defendía la distribución más amplia posible de la propiedad de los medios de producción. Para Belloc los intentos de reformar la sociedad mediante la intervención estatal, disociados de principios fundamentales y enraizados en una filosofía errónea, no consiguen sino acrecentar los problemas que tratan de resolver. De este modo, el reformador que emplea sus herramientas de planificación en aras de la mejora social acaba promoviendo la imposición de diversas formas de trabajo obligatorio, características del tipo de relaciones sociales que Belloc definió como Estado Servil.

Palabras clave: Hilaire Belloc; Estado del Bienestar; Estado Servil; colectivismo.

Clasificación JEL: B14, B15, B25

Abstract: Hilaire Belloc (1870-1953) criticized the social legislation passed by the British Liberal Party before the Great War, which represented the emergence of the modern Welfare State. His criticism was based on the ideas of a school of thought called distributism. Based upon the principles of Catholic

* Doctorando del programa de CC. Sociales y Jurídicas de la Universidad Rey Juan Carlos. Master Universitario en Humanidades por la Universidad Francisco de Vitoria. Licenciado en CC. Económicas por la Universidad de Alicante. Email: a.diazve.2016@ alumnos.urjc.es 
Social Teaching, especially the encyclical Rerum novarum. This Thomist rooted school of thought praised that the means of production should be spread as widely as possible. Belloc believed that attempts of social reform by state intervention, dissociated from fundamental principles, would lead to results opposite to those initially intended. Social reforms aimed to improve the status of workers could lead, by the needs of their sustainability, to an economy in which certain people would be forced by regulation to work for others or for the state, who likewise would have to take care of them. Belloc coined the concept "Servile State" for this kind of society.

Keywords: Hilaire Belloc; Welfare State; Servile State; collectivism

JEL codes: B14, B15, B25

Del suelo una flor levanta, Llévala y dile a Alejandro, Que digo yo, que me haga, Solo una flor como ella, Verás luego que no pasan,

Trofeos, aplausos, glorias, Lauros, triunfos y alabanzas, De lo humano; pues no puede,

Después de victorias tantas, Hacer una flor tan fácil, Que en cualquier campo se halla.

Calderón de la Barca; La cisma de Inglaterra, Jornada Segunda. 


\section{INTRODUCCIÓN}

Las palabras que Calderón de la Barca pone en boca del bufón Pasquín, quien cuenta una fábula a Enrique VIII para disuadirle de seguir los consejos del adulador Cardenal Bolseo (Wolsey), sirven para caracterizar la clase de problema al que se enfrenta el planificador económico. Ese problema no es otro que la imposibilidad de alcanzar, organizando los medios a su disposición de una manera racional, frutos mejores que los que el orden natural ofrece de manera espontánea. Del mismo modo que Alejandro Magno, conquistador de imperios, sería incapaz de crear una simple flor, que el campo regala por doquier, los diseños conscientes y deliberados del planificador racional, cargados de lógica en el mundo ideal de la abstracción, no logran, como pretenden, superar los resultados que proporciona la interacción no coordinada de múltiples planes, medios y fines personales.

Este ejemplo ilustra la distinción clásica entre procesos «naturales» y «artificiales», que restringe el ámbito de decisión de los humanos a los segundos. Esta diferenciación y esta restricción entran en crisis hacia el comienzo de la Edad Moderna, un tiempo de convulsiones y cismas en la Iglesia cristiana, de los cuales Enrique VIII y Thomas Wolsey fueron algunos de sus más controvertidos actores, y es definitivamente cuestionada con la aparición de la teoría social moderna en el denominado Siglo de las Luces. Así lo interpretaba Friedrich A. Hayek, quien era partidario de la introducción de una tercera categoría de procesos que comprendiera «todos aquellos modelos o aquellas regularidades no intencionadas que vemos existen en la acción humana y cuya explicación corresponde a la teoría social $»^{1}$. Esta categoría intermedia se situaría a medio camino entre la de los fenómenos naturales, totalmente independientes de la acción humana, y la de los artificiales, que son fruto directo de un plan humano.

Como el propio Hayek reconoce ${ }^{2}$, Luis de Molina ya reparó en esta categoría de procesos en su tratado De iustitia et iure (1593), en

\footnotetext{
${ }^{1}$ Hayek (2012), p 154

${ }^{2}$ Ibid, p 155.
} 
el que identifica los hechos económicos con aquellos sucesos que «son el resultado de la acción humana, pero no del designio humano» ${ }^{3}$. Sin embargo, el pensamiento económico posterior recibiría mucha más influencia del constructivismo racionalista de Descartes que, en opinión de Hayek, tiene su origen en una errónea omisión de esta tercera categoría de fenómenos: aquellos que, siendo fruto de la acción individual, no responden a un plan humano. En la tradición de Carl Menger, Hayek asigna a la formación de estos fenómenos espontáneos un carácter evolutivo, parecido al que la biología atribuye a los organismos, mediante la competencia entre instituciones y tradiciones alternativas, que acaba preservando las más exitosas y desplazando a las demás.

El constructivismo racionalista, en su pretensión de determinar las cuestiones sociales y económicas mediante el diseño de un plan intencionado (ignorando así la visión sobre naturaleza de las mismas de autores como Luis de Molina), peca, en palabras del propio Hayek, de una «fatal arrogancia», consistente en creer que la información y los conocimientos que poseen los planificadores, por fuerza limitados, son todos los existentes. La arrogancia parte del error intelectual que supone desconocer que esa información y esos conocimientos se generan en un proceso dinámico de naturaleza espontánea, que no puede ser sustituido por diseños conscientes de una sola mente, por brillante que ésta pudiera llegar a ser. Esta arrogancia resulta fatal por los resultados que produce en las sociedades donde es experimentada. Hayek nos advierte de los peligros del colectivismo, que van mucho más allá de lo económico, y de la incomprensión existente, que persiste en nuestros días, sobre a la naturaleza y origen de dichos peligros.

La crítica de Hayek a estos esfuerzos planificadores que, pudiendo ser bienintencionados, suelen alcanzar resultados opuestos a los que persiguen debido a un error intelectual inherente a la filosofía que los inspira, converge con la de otros autores que han tratado de analizar la naturaleza de estos procesos. Uno de ellos, Hilaire Belloc (1870-1953), atribuye el carácter contraproducente de estos esfuerzos reformadores, más que a la intencionalidad de

${ }^{3}$ Gómez Rivas (2014), p. 25. 
quienes los promueven, a la «falsa filosofía» que los inspira ${ }^{4}$. En su conocida obra Camino de servidumbre, Hayek realiza una elogiosa referencia al trabajo de Belloc:

No hace treinta años que Mr. Hilaire Belloc, en un libro (El Estado Servil, 1913) que explica más de lo que ha sucedido desde entonces en Alemania que la mayoría de las obras escritas después del acontecimiento, expuso que «el efecto de la doctrina socialista sobre la sociedad capitalista consiste en producir una tercera cosa diferente de cualquiera de sus dos progenitores: el Estado de siervos» ${ }^{5}$.

El Estado de siervos o Estado Servil sería el resultado distorsionado de la acción reformadora del planificador económico. La acción reformadora se ve motivada por la existencia de un conflicto entre los valores éticos de la sociedad, de origen cristiano, y la realidad de ésta. Pero estos esfuerzos aparentemente benéficos conducen, según Belloc y por causa de esa «falsa filosofía» que él apuntaba, a resultados radicalmente opuestos a los que inicialmente se proponían.

A diferencia de Hayek, que considera instituciones y tradiciones como el producto de un proceso evolutivo, las ideas de Belloc parten de una concepción tradicional de dichas instituciones y costumbres, propia del pensamiento tomista ${ }^{6}$, que concibe las instituciones que regulan la convivencia humana como elementos dinámicos legitimados en tanto acordes con la ley natural ${ }^{7}$. Ambas visiones difieren radicalmente de las ideas constructivistas que conciben las instituciones y la cultura como productos de una voluntad humana consciente y deliberada. Esas ideas se encuentran detrás de la filosofía del reformador que intenta, mediante la planificación, superar las instituciones ancestrales y la cultura tradicional

${ }^{4}$ Esta «falsa filosofía» consistiría en una errónea concepción de lo que es el hombre apoyada en una errónea concepción de la historia (BeLLOC, 2002, p.20). Hayek prefiere el término «error intelectual», que pone el énfasis en la cuestión del conocimiento (Hayek, 2015, p.13). Ambas críticas convergen en buscar las causas de esta errónea visión en un pensamiento moderno cuya altanería desprecia la cultura y las instituciones tradicionales.

${ }^{5}$ Hayek (2010), p.42.

${ }^{6}$ Antuñano (2004), p.279.

${ }^{7}$ Cendejas (2016), p.30. 
en aras de la mejora social. Hayek apunta, como responsables principales de esta teoría social moderna, a las ideas de Descartes y Rousseau, en tanto que Belloc pone el énfasis en la responsabilidad de las ideas y procesos asociados a la denominada Reforma Protestante como desencadenantes de lo que Eric Voegelin caracterizó como el «descarrilamiento» de la cultura occidental ${ }^{8}$.

II

HILARIE BELLOC

Joseph Hilaire Pierre René Belloc (Celle Saint Cloud, 1870-Guilford, 1953), hijo de un abogado francés y una escritora inglesa activista de los derechos de la mujer, nació durante la Guerra FrancoPrusiana. Su familia, huyendo de la convulsa situación en la que la derrota había dejado a Francia, emigró a Inglaterra, donde su padre falleció cuando Hilaire contaba tan solo dos años, y fue su madre, Elizabeth Rayner Parkes, quien se encargó de sacar adelante a sus dos hijos y darles una educación católica.

Desde su infancia, la vida de Belloc estuvo marcada por dos realidades fundamentales que imprimieron una fuerte huella en su carácter: su condición de inglés de origen francés (nunca renegó de su origen e incluso hizo el servicio militar para no perder la nacionalidad francesa); y su catolicismo.

Cursó estudios de Historia en el Balliol College de Oxford, donde destacó pronto como brillante orador en los debates de la Oxford Union, de la que fue presidente. Al terminar sus estudios, aspiró a varios puestos de profesor sin tener éxito, debido en alguna medida a su condición de católico, que se encargaba de enfatizar acudiendo a los exámenes con una pequeña imagen de la Virgen' Desarrolló una larga carrera de escritor y periodista. Alcanzó su primer éxito editorial con la novela El camino a Roma, y colaboró en distintas publicaciones, entre ellas New Age, en la que polemizó activamente con socialistas fabianos como H.G. Wells o G. B. Shaw, coincidiendo con su inseparable amigo Gilbert Keith Chesterton.

\footnotetext{
${ }^{8}$ Voegelin (1992), p.80.

9 Pearce (2015), p.66.
} 
Su mayor éxito como escritor fue el libro de poemas infantiles Cautionary Tales for Children, que sigue siendo popular en el Reino Unido.

Su espíritu inconformista y su preocupación por los asuntos de su país le llevaron a presentarse en 1906 a las elecciones al Parlamento como candidato del Partido Liberal por el distrito de Salford, de mayoría obrera. Tras una dura campaña en la que su origen francés y su catolicismo fueron utilizados por sus contrincantes como armas arrojadizas, que supo contrarrestar con su brillante oratoria, resultó elegido Miembro del Parlamento.

$\mathrm{Su}$ trayectoria como parlamentario resultó cuanto menos controvertida. Destacó por su oposición a muchas de las medidas del gobierno de su propio partido, particularmente a aquellas que tendían a regular las relaciones laborales o la conducta de los ciudadanos. Pero la opinión que más animadversión le valió dentro de su propio partido fue su oposición a la Licensing Bill y otras medidas similares destinadas a tratar de limitar el consumo de alcohol entre las clases bajas y que fueron denominadas satíricamente Teetotalism $^{10}$. Como representante de un distrito obrero, encontró intolerable tal intromisión en la vida y la libertad de los ciudadanos. Su actitud le valió no ser candidato del Partido Liberal para las siguientes elecciones. Tampoco consiguió el escaño al presentarse como independiente, por lo que en 1910 terminaría su breve y polémica carrera política.

Su paso por el Parlamento marcó sus posteriores opiniones como periodista y escritor extremadamente crítico con los vicios de una política que había conocido de primera mano. Como coeditor del Eye Witness junto con Cecil Chesterton, denunció en 1912 el conocido escándalo Marconi ${ }^{11}$, que costó a éste último una condena judicial por injurias y a ambos acusaciones de antisemitismo. La crítica revista de Cecil Chesterton, hermano de Gilbert Keith, y Belloc se refundó como New Witness en 1914. Aquel mismo año Belloc

10 Ibid, p.179.

11 El escándalo Marconi de 1912 se sustentó en acusaciones periodísticas a miembros del gobierno británico sobre la compra de acciones con información privilegiada de la filial americana de la compañía del mismo nombre. Entre los señalados se encontraban Lloyd George y Sir Rufus Isaacs, fiscal general y hermano del director de la compañía Marconi, Godfrey Isaacs. 
sufrió el durísimo golpe de la muerte de su esposa a causa de la gripe, además del comienzo de la Gran Guerra, que supuso, cuatro años más tarde, la muerte de su hijo Louis y de su amigo Cecil Chesterton. Gilbert Keith, ya convertido al catolicismo, tomó el relevo periodístico de su hermano colaborando con Belloc a partir de 1925 al frente del G.K.'s Weekly, que se convirtió en la voz del movimiento distributista. A la muerte de éste en 1936, correspondió a Belloc la dirección de la revista que pasó a denominarse The Weekly Review. A través de estas publicaciones y de sus ensayos, entre los que destaca The Servile State (1913), configuró junto con los hermanos Chesterton, Maurice Baring ${ }^{12}$ y otros las líneas generales de la corriente de pensamiento que sería conocida como distributismo, una opción alternativa a capitalismo y socialismo consistente en una sociedad libre y respetuosa con el derecho a la propiedad en la que el control de los medios de producción estuviese ampliamente distribuido.

III

\section{EL ESTADO DEL BIENESTAR}

Hilaire Belloc conoció algunas de las manifestaciones incipientes del Estado del Bienestar. Aunque el término Welfare State ve la luz durante la Segunda Guerra Mundial ${ }^{13}$, en la legislatura 1910-1914, la siguiente a la que vio a Belloc en la Cámara de los Comunes, el gobierno liberal de Asquith aprobó la Ley Nacional del Seguro (National Insurance Act, 1911) y otras normas que se consideran manifestaciones primitivas de una función asistencial del Estado que ha venido ampliándose hasta nuestros días. La Ley Nacional del Seguro establecía prestaciones para los trabajadores en situaciones

12 Maurice Baring (1874-1945), miembro del círculo de Chesterton y Belloc, fue un escritor y periodista británico, converso al catolicismo. Adquirió fama como dramaturgo y novelista tras la Primera Guerra Mundial.

${ }_{13}$ El denominado Informe Beveridge (oficialmente Social Insurance and Allied Services, http://news.bbc.co.uk/2/shared/bsp/hi/pdfs/19_07_05_beveridge.pdf) de 1942 es considerado como el documento fundacional del Estado del Bienestar moderno. Tras la guerra, la victoria del Partido Laborista en las elecciones de 1945 supuso reformas legislativas adicionales que implementaban las ideas de Beveridge. 
de enfermedad o desempleo en base a fondos aportados mediante cotización. Belloc, ya fuera del Parlamento y en su calidad de periodista y comentarista político, criticó abiertamente ésta y otras leyes del ejecutivo liberal de Asquith, centrando sus críticas fundamentalmente en el miembro del gabinete encargado de la Hacienda, Lloyd George.

Belloc consideraba esta ley como manifestación y prueba del advenimiento efectivo de lo que él denominó el Estado Servil. En su obra del mismo nombre, escrita un año después de la aprobación de la Ley Nacional del Seguro, consideró que dicha ley, en tanto que destinada a paliar la inseguridad del proletariado, «sigue las directivas de un Estado Servil en todos sus detalles $»^{14}$. Las razones que refiere para apoyar esta afirmación son:

a) Que no da cobertura a los ciudadanos en tanto que tales, sino en la medida en que son trabajadores. El criterio para aplicación de la ley es el empleo. Y no cualquier tipo de empleo, sino únicamente aquel propio del «individuo común que trabaja con sus manos», es decir, el proletariado. La ley excluía «las formas de trabajo a la que se dedican las clases educadas $»^{15}$, como el propio oficio de Belloc, el de escritor, y limitaba su aplicación tan solo al ámbito de los trabajos manuales. No es que Belloc fuese partidario de una extensión de su ámbito de aplicación, sino que veía en esta característica un reflejo de la intención del legislador.

b) Que la ley implica el reconocimiento de un estatus, que sustituye a las anteriores relaciones entre iguales basadas en un contrato. El cumplimiento de la ley, en la medida en que las aportaciones al seguro son deducidas de los salarios e ingresadas en el fisco por el empleador, queda en manos de éste, al que se le impone la obligación de «fiscalizar al proletariado». Esto supone, para Belloc, el reconocimiento legal de dos categorías de ciudadanos empujando a «los que están abajo a registrarse, pagar un impuesto (...) y a los que están arriba a que sirvan de

\footnotetext{
${ }^{14}$ Belloc (2010), p. 175.

${ }^{15}$ Ibid.
} 
agentes para hacer cumplir tal registro y recaudar tal impuesto $»^{16}$.

La división de los ciudadanos en dos clases legalmente reconocidas: capitalistas y proletarios, supone el reconocimiento de la división de la sociedad en dos grupos e impone, bajo la autoridad del Estado, una nueva institución a la sociedad. La diferenciación en base a la posesión o no de los medios de producción iba en contra de la práctica, habitual desde el derecho romano, del contrato libre entre iguales. Esta división de la ciudadanía en categorías (los económicamente libres y los económicamente menos libres), recogida en la Ley Nacional del Seguro, se acabará extendiendo, vaticina Belloc, a todos los aspectos de la economía. Belloc relacionaba el subsidio por desempleo con el concepto de salario mínimo y pronosticaba que la imposición de éste traería consigo, como término recíproco, el principio de trabajo obligatorio ${ }^{17}$.

Estas reformas constituyeron, como se ha indicado, precedentes de lo que posteriormente se conocería como Estado del Bienestar, que fue experimentado en la Inglaterra de la Segunda Guerra Mundial y posteriormente extendido durante la postguerra a los principales países industrializados de Europa y América. De acuerdo con el enfoque keynesiano, sirvió como herramienta de política económica en la crisis que siguió al gran conflicto ${ }^{18}$. La desmovilización de gran cantidad de mano de obra y recursos destinados al esfuerzo bélico, devino en la necesidad de potenciar la industria de bienes de consumo, para lo cual las prestaciones, ayudas y garantías económicas que el Estado de Bienestar desplegaba servían de estímulo mediante la promoción del consumo privado. La crisis de los 70, en la que las políticas de impulso a la demanda no resultaron eficaces frente a una recesión iniciada por causas relativas a la oferta (subida de precios de materias primas básicas) y a la crisis del modelo monetario, supuso un cuestionamiento de las políticas identificadas con el Estado del Bienestar. Dicho cuestionamiento se ha basado fundamental-

\footnotetext{
${ }^{16}$ Ibid, p. 176.

17 Belloc (2010), p. 178.

18 Picó (1999), p. 77.
} 
mente en la insostenibilidad económica de los niveles de prestaciones alcanzados, que unida a la dificultad político-sociológica de su reducción una vez implementados puede amenazar el desarrollo de la economía y por lo tanto el bienestar futuro. Las críticas al Estado del Bienestar, más allá del asunto de su sostenibilidad, tienen su fundamento en teorías que ensalzan la independencia del individuo respecto del Estado. En los años 80, se llevaron a la práctica en Estados Unidos y Gran Bretaña mediante los recortes presupuestarios de los últimos mandatos de R. Reagan y M. Thatcher $^{19}$.

En los últimos años las políticas relacionadas con el Estado del Bienestar han vuelto a verse afectadas, en los principales países desarrollados, por los efectos de la crisis económica iniciada en 2008. Sin embargo, en el plano teórico y, sobre todo, en el político y sociológico, el concepto de Estado del Bienestar no es cuestionado, al menos en el ámbito europeo, prácticamente por ninguna opción política. Las reducciones en las prestaciones, debidas a los efectos de la crisis sobre las finanzas públicas, son vistas por unos y otros como negativas, si bien los partidarios de las mismas las delatan como «mal necesario» para poder mantener las prestaciones propias del Estado del Bienestar en el futuro. Es decir, las críticas al Estado del Bienestar no guardan relación con su bondad o conveniencia, que se considera prácticamente incuestionable, sino con su sostenibilidad.

Por el contrario, Belloc criticó enfáticamente las primeras manifestaciones de este Estado del Bienestar hoy escasamente cuestionado. Para él estas reformas sociales, como recoge el ideario de sus principales promotores, los socialistas fabianos con los que Belloc solía polemizar, constituían tan solo pequeños pasos de un camino que conduce directamente hacia el colectivismo. De cómo se recorrería, en opinión de Belloc, ese camino hacia la servidumbre económica, versará su más conocido ensayo: El Estado Servil.

\footnotetext{
19 Picó (1999), p. 27.
} 
IV

\section{EL ESTADO SERVIL}

El Estado Servil (The Servile State, 1913) es considerado, junto con las obras de G.K. Chesterton Lo que está mal en el mundo (1910) y Los límites de la cordura (1926), uno de los ensayos fundamentales ${ }^{20}$ de la corriente de pensamiento conocida como distributismo ${ }^{21}$.

En su análisis general de la historia económica del mundo occidental, y en particular de Inglaterra, Belloc emplea una serie de conceptos que conviene aclarar previamente. Su definición de capitalismo no pone el énfasis, como hacen muchos autores socialistas, en la organización de la producción y el comercio por los particulares o en la existencia de la propiedad privada de los medios de producción, sino en la concentración de esta última en muy pocas manos. Al igual que otros autores distributistas, considera que lo que define a la sociedad capitalista de su tiempo es «que la propiedad de la tierra y el capital - es decir, la posesión, y por tanto el dominio de los medios de producción- está limitada a cierto número de ciudadanos libres no lo suficientemente grande como para determinar la masa social del Estado, mientras que los restantes carecen de propiedad y son, por tanto, proletarios $»^{22}$. En la misma línea, su amigo G.K. Chesterton afirmó descriptivamente que «lo que llamamos capitalismo debería llamarse proletarismo ${ }^{23}$. Y es que los autores distributistas, a la hora de criticar el capitalismo de su tiempo y buscar explicación a sus consecuencias sociales negativas, situaban en el punto de mira la concentración de la propiedad de los medios de producción.

${ }^{20}$ Otras obras de Belloc en relación al distributismo son: The Church and Socialism (1909); The Catholic Church and the Principle of Private Property (1920); y An Essay on the Restoration of Property (1936).

${ }^{21}$ Movimiento basado en la encíclica Rerum Novarum que proponía una distribución lo más amplia posible de la propiedad de los medios de producción. Más que un sistema político-económico alternativo a capitalismo y socialismo, el distributismo constituye «una concepción de la persona, de la sociedad y de la cultura que se deriva de unas formas económicas, sociales, políticas orientadas a la plenitud del ser humano» (Antuñano, 2004, p.282).

${ }^{22}$ Belloc (2010), pp. 52-53.

${ }^{23}$ Chesterton (2010), p. 20. 
Para Belloc el capitalismo no solo no implica un estado de la sociedad en el que el capital es propiedad privada de los ciudadanos, sino que considera tal sociedad como opuesta al concepto de capitalismo tal y como él lo utiliza ${ }^{24}$. A esa sociedad ideal en la que la propiedad de los medios de producción está ampliamente distribuida entre la población la denomina Estado Distributivo. En el extremo opuesto, a la sociedad ideal que niega completamente la propiedad privada y en la que «los medios de producción se encuentran en poder de los agentes políticos de la comunidad $»^{25}$ la denomina colectivista. Como complemento a estos tres tipos de sociedad que se dan en alguna medida en la realidad o a lo largo de la historia (capitalista, colectivista o distributiva), Belloc introduce una nueva definición: el Estado Servil. Es servil «aquel régimen social en que las familias y los individuos están obligados por ley a trabajar en beneficio de otras familias o individuos, en número tan considerable que imprimen sobre toda la comunidad la marca de tal género de trabajo $»^{26}$.

En su análisis histórico Belloc comienza destacando que las sociedades paganas de la Antigüedad, particularmente en el ámbito europeo, respondían al modelo que él denomina Estado Servil, en la medida en que en todas ellas era predominante el tipo de trabajo legalmente obligatorio que caracteriza a éste. Existían por tanto dos tipos de miembros de dichas sociedades: hombres libres y esclavos. La esclavitud en estas sociedades paganas (desde los pueblos germanos y celtas hasta Grecia o Roma), afirma Belloc, no tenía una condición racial, sino ante todo social. Pese a que muchos esclavos se obtenían como botín de guerra, el origen de la institución, en contra de lo que a veces se piensa, es ante todo económico. «El esclavo nació nada más que de la pobreza ${ }^{27}$, afirma un Belloc que ve en la búsqueda de una posibilidad de supervivencia frente a la indigencia la causa principal de la institución esclavista. Sería por tanto la imposibilidad de encontrar

${ }^{24}$ «Nor does Capitalism mean a state of society in which capital is owned as private property by the citizens. On the contrary, such a society of free owners is the opposite of Capitalism as the Word is used here». (Belloc, 2002, p.5).

${ }^{25}$ Belloc (2010), p. 52.

${ }^{26} \mathrm{Ibid}, \mathrm{p} .53$.

${ }^{27}$ Ibid, p. 66. 
otro medio de vida la razón por la que muchas personas aceptarían convertirse en esclavos.

Esta explicación concuerda con la de Aristóteles (Política, Libro I), que consideraba la esclavitud como inevitable y natural argumentando que hay personas que nacen para mandar y otros que lo hacen para obedecer, si bien en este caso no se da la aversión moral que Belloc y sus contemporáneos sentían hacia tal institución. La economía de las sociedades paganas, caracterizada por el recurso a la esclavitud fue evolucionando a raíz de la irrupción en la historia de lo que Belloc denomina «el experimento llamado la Iglesia de Cristo» que produjo, según él, «la transformación lenta del Estado Servil en algo distinto: una sociedad de propietarios $»^{28}$. Ese lento proceso vino caracterizado por la paulatina evolución de la esclavitud hacia la servidumbre agraria, asociada a la tierra, más que a la persona, a través de la villa romana. Posteriormente el labriego vería consolidado su derecho al uso de la tierra, quedando a su disposición el excedente de una cantidad fija que habría de entregar al propietario. Por este sistema consuetudinario el agricultor fue tomando el control, en condición de usufructuario cuando no de propietario efectivo, de la producción y de la tierra. Instituciones como las tierras de dominio público o demaniales, los monasterios o los gremios, contribuyeron a promover una sociedad que en determinadas zonas, como Inglaterra y Francia ${ }^{29}$, estuvo basada en el principio de la propiedad ${ }^{30}$.

Para describir el paso de un tipo de sociedad a otra a lo largo de la historia emplearemos un gráfico que relaciona cualitativamente

${ }^{28}$ Ibid, p. 71.

${ }^{29}$ En el caso de España se podría argumentar que también se dio, e incluso en época más temprana, una sociedad de propietarios, debido al menor impacto de la institución feudal y a la empresa de la Repoblación de las tierras reconquistadas. Las necesidades de este proceso implicaban el avance de colonos libres que poseían las tierras que repoblaban.

30 «Tal fue la transformación que había sobrevenido en la sociedad europea en el curso de diez siglos de cristianismo. La esclavitud había desaparecido, y en su lugar había surgido ese establecimiento de la posesión libre que parecía tan normal a los hombres y tan apropiado para una vida feliz. No se encontró a la sazón nombre especial alguno que la denominara. Hoy en día, es decir, cuando ha desaparecido, debemos fabricar uno torpemente, y decir que la Edad Media había concebido instintivamente y engendrado el Estado Distributivo» (Belloc, 2010, p.82). 
dos conceptos que para Belloc caracterizan estas tipologías: la distribución de la propiedad (de los medios de producción) y la libertad (tanto económica como política). Para Belloc ambos conceptos están directamente relacionados, de modo que la sociedad pagana de la Antigüedad evoluciona de manera estable hacia una sociedad de hombres libres en la medida en que una propiedad (en forma de tierra o de derechos sobre la misma) ampliamente distribuida pasa a marcar el «tono» de dicha sociedad. Ordenando sobre un eje ambos sistemas en función de su mayor o menor grado de libertad y distribución de la propiedad (Figura I) caracterizamos gráficamente el paso de una sociedad esclavista a una de propietarios descrito por Belloc en las secciones II y III de El Estado Servil.

FIGURA I

DEL ESTADO SERVIL AL ESTADO DISTRIBUTIVO

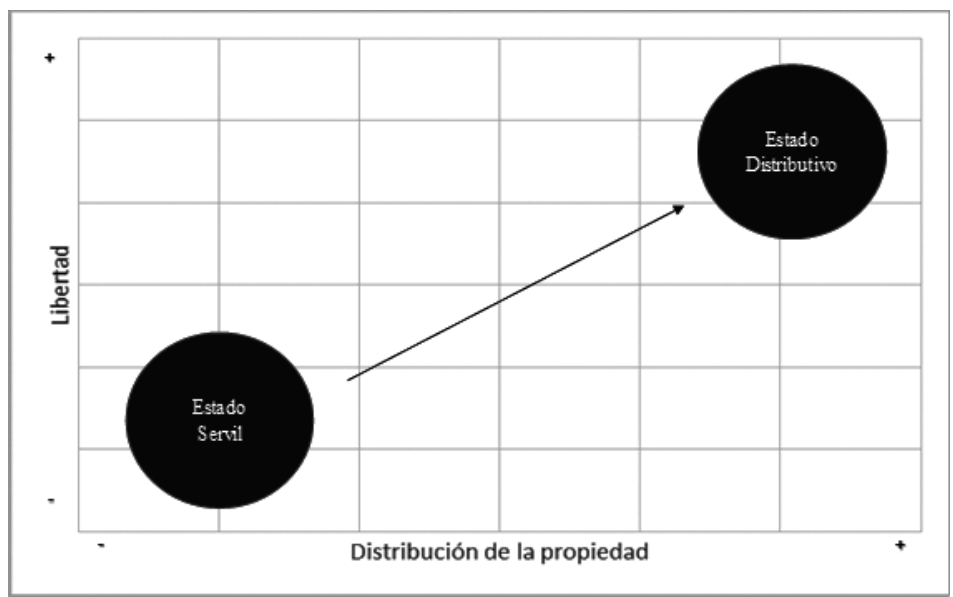

Aquel sistema que Belloc describió como Estado Distributivo y que correspondería fundamentalmente al régimen de explotación de la tierra en la sociedad europea tardo-medieval, vino a malograrse en muchos lugares, particularmente en Inglaterra, a partir del siglo XVI. Belloc describe el desmantelamiento del Estado Distributivo como un proceso en el que la concentración de la propiedad de la tierra en pocas manos (desde las confiscaciones de Enrique 
VIII hasta los Inclosure Acts del siglo xVIII) y la eliminación de instituciones que servían de barrera para el surgimiento de monopolios imponen paulatinamente el control de la economía por parte de una reducida oligarquía. La explicación según la cual la concentración de la propiedad sobreviene de manera natural como consecuencia de la Revolución Industrial es denostada por Belloc, quien afirma que «ninguna causa material de tal género determinó la degradación que padecemos» ${ }^{31}$.

FIGURA II

DEL ESTADO DISTRIBUTIVO A LA ECONOMÍA MODERNA, CARACTERIZADA POR UNA FUERTE CONCENTRACIÓN DE LA PROPIEDAD

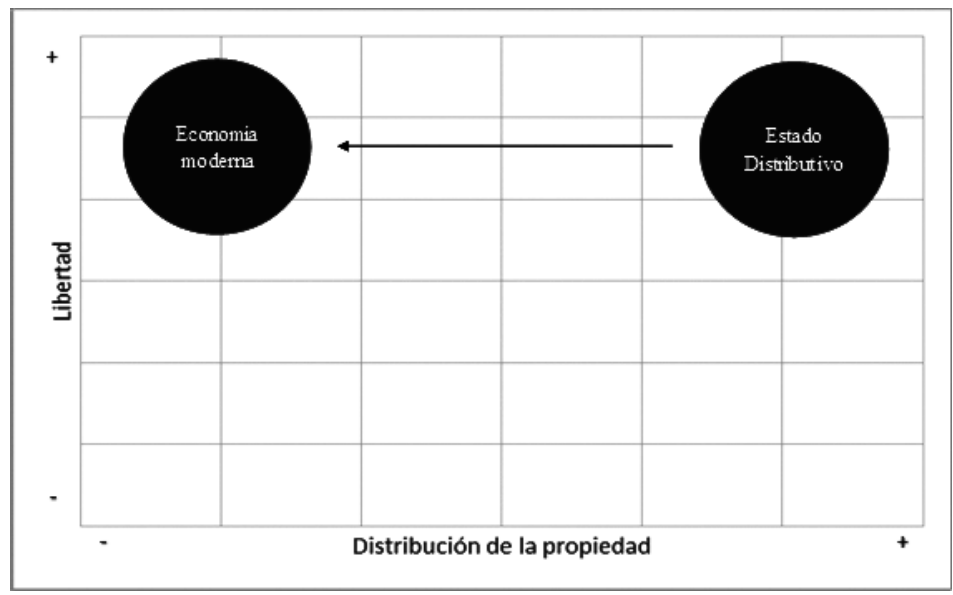

El tipo de economía a la que dio lugar el proceso descrito por Belloc se encuentra, en su opinión, en una posición de desequilibrio (Figura III), debido a la combinación entre libertad nominal y negación del acceso a la propiedad de la mayoría de personas. Para Belloc un sistema se encontraría en posición de equilibrio en la medida en que libertad y propiedad fuesen de la mano, pues para él no pueden darse de manera estable la una sin la otra, dado que «solo mediante

${ }^{31}$ Belloc (2010), p. 82. 
una propiedad bien dividida pueden las unidades de la sociedad reaccionar frente al Estado» ${ }^{32}$. La libertad económica, otorgada por la propiedad, está, para Belloc, estrechamente relacionada con la política. De este desequilibrio resultarían dos tensiones internas:

a) El conflicto entre la realidad social y su base moral, pues esta última se encuentra aún impregnada por el cristianismo. Instituciones como la esclavitud o la servidumbre cuentan con el rechazo generalizado de la sociedad, como también lo hacen la pobreza y las condiciones indignas de vida, de modo que «la vida real de la sociedad se encuentra divorciada del fundamento moral de sus instituciones» ${ }^{33}$.

b) La inseguridad y la penuria a la que ciudadanos nominalmente libres se veían avocados, de modo que el propio capitalismo debía proveer soluciones no capitalistas a la situación, como por ejemplo las sucesivas leyes sobre pobreza (Poor Relief Acts), entre las que destacó la de 1834, vigente durante la era victoriana.

FIGURA III

LA ECONOMÍA MODERNA, CON SUS TENDENCIAS

CONTRADICTORIAS, SE ENCUENTRA EN DESEQUILIBRIO

EN RELACIÓN AL BINOMIO PROPIEDAD-LIBERTAD

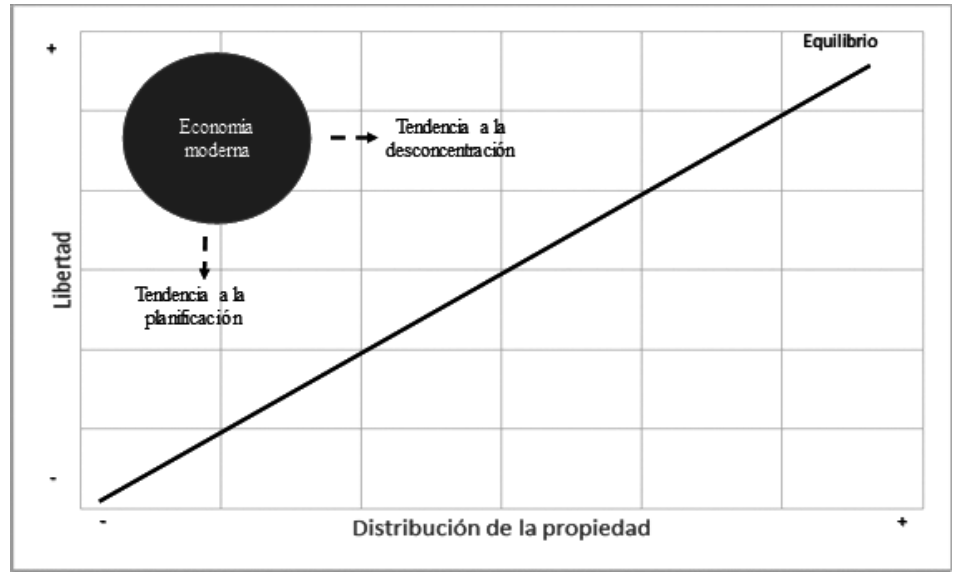

32 «Through well-divided property alone can the units of society react upon the State» (Belloc, 2002, p.10).

${ }^{33}$ Belloc (2010), p. 110. 
Estas tensiones podrían por tanto solucionarse mediante dos vías o tendencias contrapuestas: tratando de incrementar la distribución de la propiedad de los ciudadanos (opción que hemos denominado «tendencia a la desconcentración» en la figura III); o adoptando medidas reformadoras que impliquen la acción de los poderes públicos tratando de paliar los efectos de la ausencia de ésta («tendencia a la planificación»). Cada una de estas opciones caracteriza una de las soluciones al problema del desequilibrio entre propiedad y libertad.

FIGURA IV

LA ECONOMÍA MODERNA DEBE EVOLUCIONAR

A ALGUNA DE LAS POSICIONES DE EQUILIBRIO DEL BINOMIO PROPIEDAD-LIBERTAD

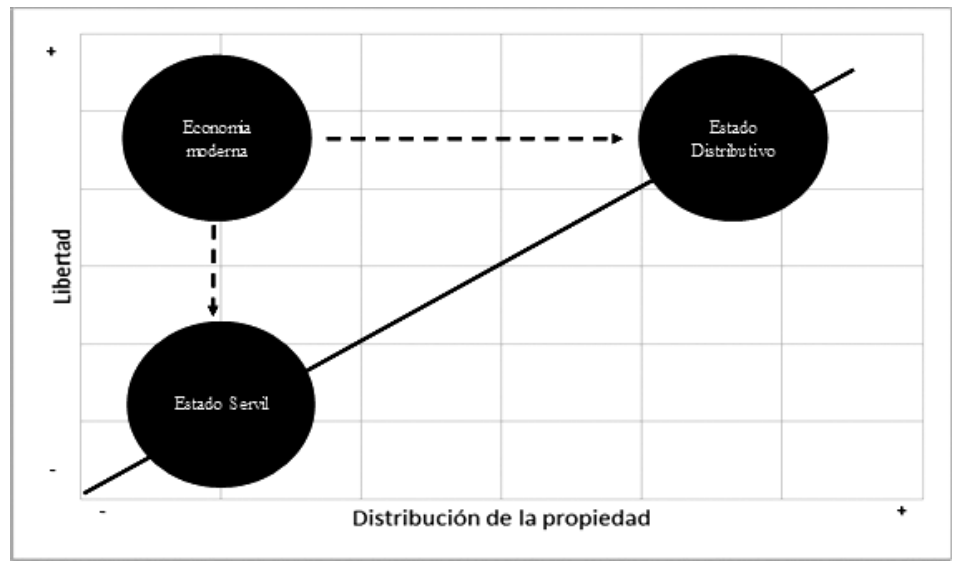

La vuelta al equilibrio en relación a este binomio propiedad-libertad (Figura IV), ha de pasar, por tanto, por uno de estos dos caminos:

a) Procurando una amplia distribución de la propiedad de los medios de producción entre los ciudadanos considerados nominalmente libres ${ }^{34}$.

${ }^{34}$ Belloc no establece en «El Estado Servil» un programa claro respecto a cómo se podría alcanzar este propósito. En otra obra (Belloc, 2002, pp. 31-66) sugiere un progra- 
b) Limitando la libertad de los ciudadanos que no tienen acceso a la propiedad de manera que deban trabajar obligatoriamente.

Ambas soluciones se corresponden, respectivamente, con los modelos «de equilibrio» descritos por Belloc como Estado Distributivo y Estado Servil (Figura IV). En El Estado Servil, escrita cuando aún no había antecedentes de experimentos comunistas a gran escala, considera el colectivismo como una consecuencia natural de la ideología materialista inherente al capitalismo, de modo que "el Estado capitalista engendra una teoría colectivista que, al aplicarse, produce algo completamente distinto del colectivismo, a saber: el Estado Servil" ${ }^{\prime \prime 35}$. Posteriormente pasaría a considerar el capitalismo de su época y el comunismo real ya ensayado como dos caminos alternativos hacia un mismo resultado ${ }^{36}$. En el primero de los caminos, el más verosímil para Belloc, los reformadores socialistas actuarían paulatinamente sobre una economía capitalista, sin negar inicialmente sus fundamentos, para tratar de mejorar las condiciones sociales. En el segundo, el colectivismo prometería garantizar las condiciones para proveer bienes materiales en abundancia para todos los ciudadanos, pero solo si éstos renuncian a su libertad económica, aceptando leyes coercitivas que implican la eliminación efectiva de la propiedad privada o del derecho a la herencia ${ }^{37}$.

Para Belloc tanto el reformador socialista, ya lo sea por deseo de mejorar las condiciones de los desposeídos o por convicción en las

ma basado en la regulación, más que en la intervención estatal directa, para favorecer de manera paulatina la adquisición de pequeñas propiedades y su salvaguarda frente a procesos de concentración.

35 Belloc (2010), p. 121.

36 «To stablish the Servile State one has but to follow certain lines which lead rapidly to an ideal conclusion, a society where all men, the few Capitalists and the mass of proletariat are all securely nourished - the latter on a wage or, lacking this, a subsidy in illness. The same is true in regard to the Communist State: a society where all men are securely nourished as slaves of the government. A simple formula and its exact application will, in each case, produce the ideal society envisaged» (Belloc, 2002, p.11).

37 «Under the Communist scheme the matter is simpler still. It being made an offence for any man to own, all right to the use of accumulation by a family or an individual being destroyed, and all right of inheritance being also destroyed, the whole produce of the community is available for distribution to all. And Economic Freedom has disappeared for all though the action of a very few and simple but absolute coercive laws» (Belloc, 2002, p.12). 
bondades de la gestión centralizada, como el «hombre práctico», que pretende mejorar las condiciones «prácticas» sin atender a principios fundamentales, encuentran todos sus esfuerzos reformadores involuntariamente encauzados hacia el advenimiento del Estado Servil. Todos estos reformadores se esfuerzan por establecer medidas prácticas que los reformados (la gran masa de asalariados) agradecen, pues contribuyen a mejorar sus condiciones de vida a corto plazo. Sin embargo, estas mejoras de diversa índole, para ser sostenibles a medio y largo plazo, han de estar vinculadas a la obligación de trabajar, incrementando el miedo a perder el empleo y con él todas las ventajas adicionales que reporta. Esta obligación práctica de trabajar como asalariado, olvidando las otras opciones posibles (no trabajar $\mathrm{u}$ ordenar por cuenta propia medios de producción disponibles ${ }^{38}$ ), se constituye en la única elección posible para una mayoría de individuos. De esta forma, la tendencia hacia la planificación económica termina produciendo formas de trabajo obligatorio, al tiempo que poco motivadoras para el trabajador, alejando a la gran mayoría de ciudadanos del ejercicio de la función empresarial ${ }^{39}$ y agotando así la vitalidad económica de la sociedad. Adicionalmente, el tamaño del Estado que conlleva este proceso, y por tanto de la presión impositiva, junto con un exceso de regulación del mercado de trabajo, produce efectos depresivos sobre las dinámicas de crecimiento económico, que son, en última instancia, las mejores herramientas para la reducción de la exclusión social y la incorporación de los pobres a mejores empleos ${ }^{40}$.

38 Piénsese, en el caso español, en el desincentivo que para el trabajo por cuenta propia supone el volumen de cotización a la Seguridad Social que debería realizar trabajador autónomo para obtener una pensión similar a la de un asalariado, así como las cantidades mínimas que debe cotizar con independencia de sus ingresos.

39 En este contexto nos referimos a la función específica de ordenación de los factores de producción. En un sentido más amplio, la función empresarial puede entenderse ejercida por «cualquier persona que actúa para modificar el presente y conseguir sus objetivos en el futuro» (Huerta de Soto, 2015, p.41).

40 Añádanse las consecuencias derivadas de los efectos macroeconómicos de los abultados déficits fiscales, por vías como la inflación y la disponibilidad de crédito. 


\section{CONCLUSIONES}

El principio fundamental en el que se basa el pensamiento económico de Belloc es la distribución más amplia posible de la propiedad de los medios de producción. Cualquier intento de distribución más equitativa de la renta, sin atacar el problema de la propiedad, es decir, de obtener los beneficios propios de la actividad económica sin abordar la responsabilidad de ordenar en alguna medida sus factores, chocaría con un principio económico insalvable: la necesidad de la ganancia ${ }^{41}$.

En opinión de Belloc, para evitar que se pongan en peligro las prestaciones propias de un Estado del Bienestar, los poderes públicos deben, como contrapartida, evitar que personas libres pongan en peligro estos planes negándose a trabajar. Reformadores bienintencionados tratarán de maximizar las prestaciones y seguridades de perciben los ciudadanos. Puesto que dichas prestaciones no se financian de la nada, sino mediante impuestos que dependen de la generación de valor añadido, su continuo incremento supondrá por fuerza un endurecimiento de los requisitos para el acceso a las mismas, así como de las condiciones del propio trabajo, que han de ser cada vez más duras. Es decir, la imposibilidad de sostener económicamente dichas reformas obligará a vincularlas a formas de trabajo de hecho (aunque no necesariamente de derecho) obligatorias. De este modo se incidirá en la consolidación de dos tipos de ciudadanos nominalmente libres: una minoría, poseedora o controladora de los medios de producción, y la gran mayoría, obligada a trabajar para poder sostener las prestaciones que el Estado facilita. Esto equivaldría a la práctica desaparición de las denominadas «clases medias» en las sociedades desarrolladas.

Esta línea de pensamiento crítica en relación con la provisión pública de bienestar por el Estado, sus fundamentos filosóficos y

${ }^{41}$ «La ganancia sigue siendo una necesidad. Si se destruyera, más aún, si la ley impusiera una pérdida, tal cosa se hallaría en contradicción con el espíritu íntegro que inspiró todas esas reformas, las cuales se emprendieron con el objeto de implantar la estabilidad donde hoy impera la inestabilidad y de conciliar, como dice la irónica frase, los intereses del capital y del trabajo» (Belloc, 2010, p.186). 
sus consecuencias, no es exclusiva de Belloc. Otros autores, desde posiciones generalmente cristianas, han sostenido teorías parecidas. Wilhelm Röpke, uno de los padres de la economía social de mercado, consideró que «el auxilio prestado a las masas por el Estado es simplemente la muleta de una sociedad lisiada por el proletarismo, adaptada a la inmadurez económica y moral de las clases que emergieron de la descomposición del viejo orden social» ${ }^{42}$. Belloc atisbó ya en la sociedad de su tiempo visos de esa inmadurez económica al afirmar que los trabajadores de la Inglaterra que él conoció, habiendo olvidado ya la experiencia de la propiedad, concebían como natural su condición de asalariados ${ }^{43}$.

Eric Voegelin escribió contra el fundamento ideológico que se esconde tras la promesa de la provisión estatal de las necesidades vitales del individuo. Para Voegelin, el gnosticismo ha impreso su huella en la mentalidad moderna, de modo que el hombre de nuestro tiempo rechaza las limitaciones del ser humano y, mediante el proceso que él denominó «inmanentización de la escatología», concibe ideologías que anuncian el paraíso en la tierra. Este gnosticismo inmanentista moderno, que trata de organizar la civilización como un imperio bien regulado, conduce, según Voegelin, al totalitarismo, que es la forma final de la civilización progresista ${ }^{44}$. $\mathrm{Al}$ igual que Voegelin, Belloc también advirtió la influencia del gnosticismo de la Antigüedad en el pensamiento del mundo moderno, y concretamente en el tipo de mentalidad que caracterizaría el desarrollo del capitalismo industrial. El puritanismo, cuya ética estaba para Weber en el origen de lo que él denominó «espíritu del capitalismo» ${ }^{45}$, no

\footnotetext{
${ }^{42}$ Röpke (2014), p. 154.
}

43 «En resumen: la actitud actual del proletariado en Inglaterra (dicho de otra manera, la actitud de la inmensa mayoría de las familias inglesas) respecto a la propiedad y a aquella libertad que sólo puede obtenerse mediante la propiedad ha dejado de ser una actitud de experiencia o de expectación. Se consideran a sí mismos como asalariados, y el aumento del estipendio semanal de los asalariados es un objetivo que aprecian y persiguen intensamente; en cambio, el de la liberación de su condición de asalariados les parecería enteramente al margen de la realidad de la vida» (Belloc, 2010, p.150).

${ }^{44}$ Voegelin (1992), p. 132.

45 «The Puritan wanted to work in a calling; we are forced to do so. For when asceticism was carried out of monastic cells into everyday life, and began to dominate 
era para Belloc sino una versión actualizada de la herejía gnóstica del maniqueísmo ${ }^{46}$.

Belloc pronosticó el advenimiento del Estado Servil sobre la base de la necesidad de reducir y eliminar las tensiones que produce en la sociedad capitalista la coexistencia de un nivel elevado de libertad y una fuerte concentración de la propiedad. Dichas tensiones se ponen de manifiesto en las contradicciones entre la realidad social y los valores éticos de la sociedad, basados en unos fundamentos religiosos que se encuentran en crisis. Estas contradicciones dan lugar a su vez a un esfuerzo reformador, generalmente bienintencionado, que trata de mitigar efectos negativos como la miseria o la inseguridad. No de forma deliberada, sino como consecuencia de la aplicación de una «falsa filosofía» ${ }^{47}$, estos esfuerzos reformadores apuntalan la distribución y el control desigual de los medios de producción, pues, por fuerza de la necesidad de su sostenimiento, las prestaciones y servicios propios del Estado del Bienestar terminan imponiendo al ciudadano común la obligación práctica de trabajar para otros. De este modo la comunidad se asentará «en aquel principio servil que fue su fundamento antes de la llegada de la Fe cristiana, principio del cual esta Fe la emancipó lentamente, y al cual vuelve naturalmente por la decadencia de ésta». ${ }^{48}$

worldly morality, it did its part in building the tremendous cosmos of the modern economic order» (Weber, 2001, p. 123).

46 «To be a Puritan is almost exactly the same as to be what the old world used to call a Manichaean. The Puritan and the Manichee have the same attitude towards the universe; their creeds work out to the same moral and social practice» (Belloc, 1992, p.174).

47 «This last argument is one of the many which we find in common to those who defend the Capitalist system and those who defend the Communist system: for Socialism and Capitalism are twin successive products of the same false philosophy» (Belloc, 2002, p.32).

48 Belloc (2010), p. 191. 


\section{REFERENCIAS BIBLIOGRÁFICAS}

ANTUÑANO, S. (2004): «El distributismo inglés: una alternativa de raíz tomista a los totalitarismos del siglo XX». Cuadernos de pensamiento, Vol. 16, pp. 277-300.

Aristóteles (1988): Política, traducción de M. García Valdés. Madrid. Gredos.

Belloc, H. (1945): El Estado Servil. El Buey Mudo (2010).

- (1992): Characters of the Reformation. Rockford IL. TAN Books and Publishers.

- (2002): An essay on the Restoration of Property. Norfolk VA. IHS Press.

- (2008): Europa y la Fe. Madrid. Ciudadela Libros.

BriggS, A. (1961): «The Welfare State in Historical Perspective». European Journal of Sociology, Vol. 2(2), pp. 221-258.

Cendejas, J. L. (2016): «Ordenación de la actividad económica, ley natural y justicia en Aristóteles y en Santo Tomás», Munich Personal RePec Archive Paper, No. 73585. https://mpra.ub.unimuenchen.de/73585/

Chesterton, G.K. (2010): Los límites de la cordura. Madrid. El Buey Mudo.

- (2006): Lo que está mal en el mundo. Madrid. Ciudadela.

Gómez Rivas, L. (2014): Historia del Pensamiento Económico. Ávila. Servicio de Publicaciones de la Universidad Católica de Ávila. HAYeK, F. (2010): Camino de servidumbre. Madrid. Alianza Editorial.

- (2012): Estudios de filosofía, política y economía. Madrid. Unión Editorial.

- (2015): La fatal arrogancia. Madrid. Unión Editorial.

Huerta DE Soto, J. (2015): Socialismo, cálculo económico y función empresarial. Madrid. Unión Editorial.

PeARCE, J. (2015): Old Thunder: A life of Hilaire Belloc. Charlotte NC. Tan Books.

Picó, J. (1999): Teorías sobre el Estado del Bienestar. Madrid. Siglo XXI.

RÖPKE, W. (2014): A Humane Economy. Wilmington DW. ISI Books. SADA, D. (2005): Gilbert Keith Chesterton y el distributismo inglés en el primer tercio del siglo XX. Madrid. Fundación Universitaria. 
Voegelin, E. (1992): The New Science of Politics. Chicago. The University of Chicago Press.

Weber, M. (2001): The Protestant Ethics and the Spirit of Capitalism. Londres. Routledge Classics. 
